\title{
Role of miR-520b in non-small cell lung cancer
}

\author{
LINLIN ZHANG ${ }^{1}$ and SHUANGQUAN YU ${ }^{2}$ \\ ${ }^{1}$ Respiratory Department, Shandong Chest Hospital; ${ }^{2}$ Department of General Surgery, \\ Jinan No. 5 People's Hospital, Jinan, Shandong 250000, P.R. China
}

Received November 2, 2017; Accepted May 11, 2018

DOI: $10.3892 /$ etm.2018.6732

\begin{abstract}
The aim of the present study was to investigate the expression of microRNA (miR)-520b in non-small cell lung cancer (NSCLC) and its biological functions. Reverse transcription-quantitative polymerase chain reaction was used to detect the expression of miR-520b in 52 cases of NSCLC tissues, and its associations with tumor clinical staging and lymph node metastasis were analyzed. miR-520b mimics was transfected into A549 and Calu-3 cells. Cell proliferation, cell cycle, and cell invasion and migration abilities were assessed via cell counting kit- 8 assay, flow cytometry and Transwell chamber assay, respectively. Western blot analysis was performed to detected protein expression levels, and dual luciferase reporter assay was used to detect the gene interaction. miR-520b expression was significantly downregulated in NSCLC. The expression of miR-520b in tumor tissues at N1 stage was lower than that at the N0 stage. miR-520b expression was negatively associated with clinical TNM staging. Furthermore, miR-520b mimic transfection inhibited the proliferation and invasion and metastasis abilities of A549 and Calu-3 cells. The expression of Rab22A was downregulated in the miR-520b mimics-transfected cells, whereas E-cadherin expression was increased, and vimentin expression was downregulated. Dual luciferase reporter assay demonstrated that miR-520b directly targeted the expression of Rab22A. Furthermore, Rab22A reversal downregulated the inhibitory effect of miR-520b. miR-520b expression was downregulated in NSCLC, which was negatively correlated with lymph node metastasis and TNM staging. miR-520b targeted on Rab22A to work as a tumor suppressor, inhibiting tumor proliferation and metastasis.
\end{abstract}

\section{Introduction}

Lung cancer is one of the most malignant tumors in the world, for which there is still no effective treatment (1). In China,

Correspondence to: Miss Linlin Zhang, Respiratory Department, Shandong Chest Hospital, 46 Lishan Road, Jinan, Shandong 250000, P.R. China

E-mail: zllbbl@163.com

Key words: non-small cell lung cancer, microRNA-520b, Rab22A, tumor suppressor gene the morbidity (20.48\%) and mortality (40.71\%) of lung cancer rank as the first among all malignant tumors, representing a great threat to public health (2). Studies have demonstrated that non-small cell lung cancer (NSCLC), as the main clinical pathological type, accounts for $\sim 90 \%$ of lung cancer cases (3-5). Due to the difficulty for the early diagnosis of NSCLC, distant metastasis occurs in $\sim 40 \%$ of patients at the first diagnosis, whereas $50 \%$ of patients in the treatment process suffer from tumor metastasis (6). It has been demonstrated that, when the patients of NSCLC without distant metastasis receive appropriate treatment, the five-year survival rate may be $50-70 \%$, whereas the survival rate for the patients with metastasis would be $<5 \%$ (7). A growing body of evidence has demonstrated that tumor recurrence and metastasis represent key factors affecting the efficacy of the clinical treatment for NSCLC $(8,9)$. However, the underlying molecular mechanism has yet to be elucidated.

Recurrence and metastasis of NSCLC are closely associated with the imbalanced expression between oncogenes and tumor suppressor genes (10). When oncogenes are obviously activated and tumor suppressor genes are inactivated, NSCLC cells exhibit severe malignancy, which would gain the characteristics of metastasis, drug resistance, and self-renewal capacity $(11,12)$. Accordingly, in recent years, there has been intensive investigation focusing on the expression regulation of oncogenes and tumor suppressor genes in NSCLC, and there have been important breakthroughs (13). It has been demonstrated that epidermal growth factor receptor and Kras gene mutations can be used as predictors for the disease prognosis and the responses to molecular targeting therapies $(14,15)$. Furthermore, patients with BRAF mutations are more suitable for the target therapy with tyrosinase inhibitors (16). Furthermore, the expression of oncogene Met has important clinical value in assessing the prognosis of patients with NSCLC (17). However, at present, there is little knowledge regarding the oncogenes and tumor suppressor genes in NSCLC. Therefore, it is of great clinical importance to investigate the associated regulatory mechanisms for these oncogenes and tumor suppressor genes.

microRNAs (miRNAs or miRs), with the length of 18-22 nucleotides, are important regulatory factors for gene post-transcription, which bind to the mRNA 3'-untranslated region (UTR) to inhibit the mRNA translation (18). Studies have indicated the abnormal expression spectrum of the miR molecules in tumor tissues, including a variety of miRNAs with tumor promoting or suppressing functions, which are 
associated with the regulation of tumor cell proliferation, invasion and metastasis, angiogenesis, self-renewal capacity, drug resistance, and apoptotic processes $(19,20)$. miR-520b is a tumor-related miRNA molecule discovered in recent years, which serves the role as tumor suppressor by targeting different genes associated with the regulation of tumor proliferation, metastasis, tumor stemness, drug resistance, autophagy and immune escape. miR-520b has represented a potential target for clinical nucleic acid therapy (21). However, at present, the expression and role of miR-520b in NSCLC remains unclear. In the present study, the association between miR-520b and NSCLC pathogenesis was investigated at both the tissue and cellular levels.

\section{Materials and methods}

Tissue sample collection. NSCLC tissue samples and adjacent healthy tissue samples were collected from 52 patients with NSCLC, 29 males and 23 females, with a mean age of 46.7 years old (ranging from 24-76 years), who were diagnosed in Shandong Chest Hospital (Jinan, China) from January 2015-December 2016. Following resection, these tissue samples were washed with pre-cold saline and stored in liquid nitrogen. According to the pathological type, there were 35 cases of adenocarcinoma, 13 cases of squamous cell carcinoma and 4 cases of adenosquamous carcinoma. Based on the lymph node metastasis status, these cases were divided into the no metastasis (N0) group, the lymph node metastasis (N1) group $(n=39)$ and the non-lymph node metastasis (N2) group $(n=13)$. TNM staging (22) indicated 21 cases of stage I, 18 cases of stage II, and 13 cases of stage III. None of these patients had received any radiotherapy and/or chemotherapy, or other anti-tumor drug treatment, prior to surgical resection. Prior written and informed consent were obtained from every patient, and the study was approved by the Ethics Review Board of Shandong Chest Hospital (Jinan, China).

Cell lines and cell culture. NSCLC A549 and Calu-3 cell lines (Type Culture Collection of the Chinese Academy of Sciences, Shanghai, China) were cultured in RPMI-1640 complete medium (Gibco; Thermo Fisher Scientific, Inc., Waltham, MA, USA) at $37^{\circ} \mathrm{C}$ and $5 \% \mathrm{CO}_{2}$. Culture medium was changed every other day. Cells were passaged when $80-90 \%$ confluence was reached, and cells from passages 3-6 were used for investigation.

Reverse transcription-quantitative polymerase chain reaction (RT-qPCR). Expression levels of miR-520b in the tissues and transfected cells were detected via RT-qPCR. A total of $100 \mathrm{mg}$ fresh tissue was frozen with liquid nitrogen and ground into powder. Total RNA was extracted from the ground tissues, and A549 and Calu-3 cell lines using TRIzol (Invitrogen; Thermo Fisher Scientific, Inc.). The cDNA template was obtained through reverse transcription with the miScript II RT kit (Qiagen GmbH, Hilden, Germany), according to the manufacturer's protocol. qPCR was performed with the miScript $\mathrm{SYBR}^{\circledR}$-Green PCR Kit (Qiagen $\mathrm{GmbH}$ ) on the ABI Step one plus system (Applied Biosystems; Thermo Fisher Scientific, Inc.). The reaction system consisted of $10 \mu \mathrm{l}$ RTq-PCR-Mix,

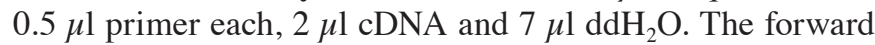

primer sequence for miR-520b was 5'-AAAGTGCTTCCT TTTAGAGGG-3', and a general primer was used for reverse primer (U6 forward, 5'-CTCGCTTCGGCAGCACA-3' and reverse, 5'-AACGCTTCACGAATTTGCGT-3'; provided within the kit). Reaction conditions were as follows: Initial denaturation at $95^{\circ} \mathrm{C}$ for $10 \mathrm{~min}$, followed by 40 cycles of denaturation at $95^{\circ} \mathrm{C}$ for $1 \mathrm{~min}$, and annealing and elongation at $60^{\circ} \mathrm{C}$ for $30 \mathrm{sec}$. The target gene expression was determined with the $2^{-\Delta \Delta \mathrm{Ct}}$ method (23).

Cell transfection. For cell transaction, $2 \times 10^{5}$ cells were seeded onto the 24-well plates. These cells were cultured with $500 \mu \mathrm{l}$ antibiotic-free RPMI 1640 medium containing $10 \%$ fetal bovine serum (FBS; Gibco; Thermo Fisher Scientific, Inc.), in a $37^{\circ} \mathrm{C}$, $5 \% \mathrm{CO}_{2}$ incubator, overnight. Cell transfection was performed when $70 \%$ confluence was reached. Briefly, $1.5 \mu \mathrm{lmiR}-520 \mathrm{~b}$ mimics (5'-AAAGTGCTTCCTTTTAGAGGG-3'; 20 pmol/ $\mu 1$; Hanheng Biotechnology Company, Shanghai, China) and $1 \mu \mathrm{l}$ Lipofectamine 2000 (Thermo Fisher Scientific, Inc.) were added to an Eppendorf tube containing $50 \mu 1$ OptiMemi medium (Thermo Fisher Scientific, Inc.). The normal control (NC) group was transfected with $20 \mathrm{pmol} / \mu \mathrm{l}$ of a nonsense miRNA sequence (5'-UCACAACCUCCUAGAAAGAGUAGA-3'; Guangzhou RiboBio Co., Ltd., Guangzhou, China). Following incubation at room temperature for $5 \mathrm{~min}$, the solution was mixed together, which, following another $20 \mathrm{~min}$, was used to incubate the cells at $37^{\circ} \mathrm{C}$ for $6 \mathrm{~h}$. Following culture with RPMI 1640 medium containing $10 \% \mathrm{FBS}$ for $48 \mathrm{~h}$ in a $37^{\circ} \mathrm{C}$, $5 \% \mathrm{CO}_{2}$ incubator, these cells were collected and prepared for the following experiments. For the transfection with Rab22A lentivirus, Lv-GFP-Rab22A lentivirus (Hanheng Biotechnology Company) was added to incubate the adhered cells at $60 \%$ confluence (at the rate of multiplicity of infection=20) at $37^{\circ} \mathrm{C}$ for $12 \mathrm{~h}$. Subsequently, the culture medium was replaced with RPMI 1640 medium containing 10\% FBS.

Cell counting kit-8 (CCK-8) assay. Cell proliferation was assessed with the CCK-8 assay (Beyotime Institute of Biotechnology, Haimen, China). Cells were seeded onto 96-well plates at a density of $2 \times 10^{3}$ cells/well. A total of $20 \mu \mathrm{l}$ CCK-8 (5 g/l) was added into the wells for incubation times of $0,24,48$ and $72 \mathrm{~h}$, at $37^{\circ} \mathrm{C}$. On the last day, $150 \mu \mathrm{lCCK}-8$ was added to the cells at $37^{\circ} \mathrm{C}$ for $2 \mathrm{~h}$. Then the absorption at $490 \mathrm{~nm}\left(\mathrm{OD}_{490 \mathrm{~nm}}\right)$ was measured for each well, and cell proliferation curves were plotted accordingly.

Transwell chamber assay. Cell invasion and migration were assessed with the Transwell chamber assay (Corning Incorporated, Corning, NY, USA). Matrigel matrix was stored in a $4^{\circ} \mathrm{C}$ refrigerator overnight. Following dilution with serum-free RPMI 1640 medium at a ratio of $1: 3$, the matrix was evenly smeared onto the upper Transwell chamber, which was subsequently incubated at $37^{\circ} \mathrm{C}$ for $60 \mathrm{~min}$. Subsequently, $1 \times 10^{5}$ cells were seeded into the upper chamber with $200 \mu 1$ serum-free medium, whereas the lower chamber was seeded with $500 \mu$ l RPMI 1640 medium containing 10\% FBS. Following incubation at $37^{\circ} \mathrm{C}$ for $24 \mathrm{~h}$, the cells were fixed with $4 \%$ formaldehyde at room temperature for $10 \mathrm{~min}$. The cells were stained using the $\mathrm{ABC}$ solution from the kit for 2 min at room temperature. Following washing, cell invasion was 
A

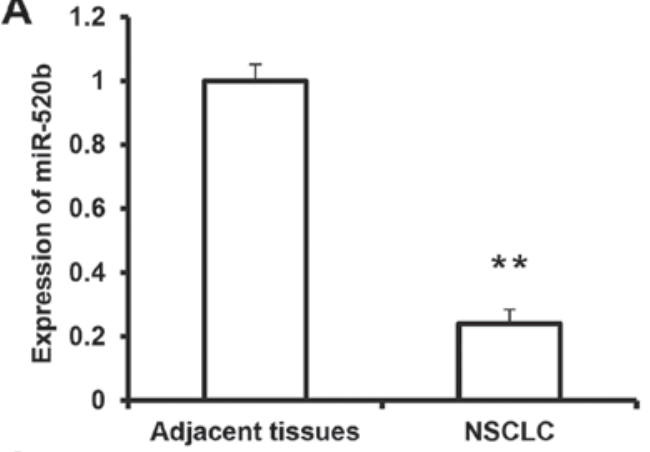

C

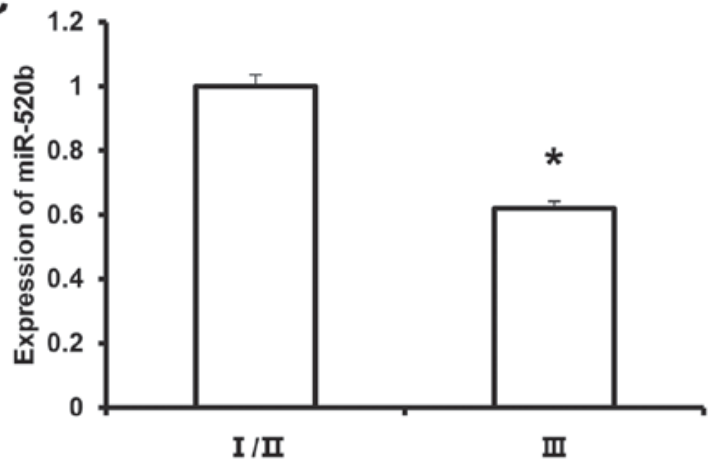

B

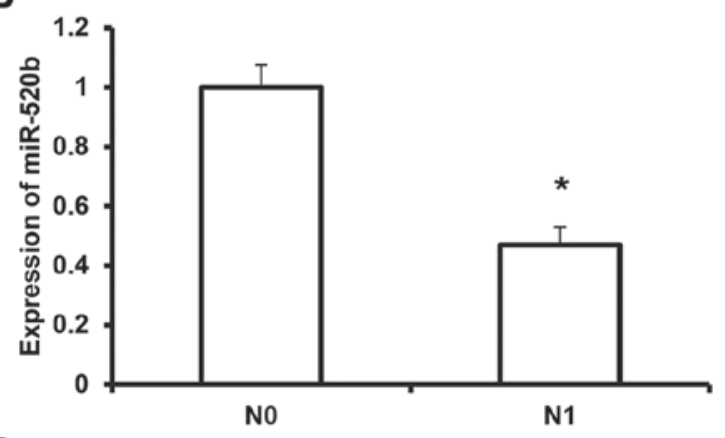

D

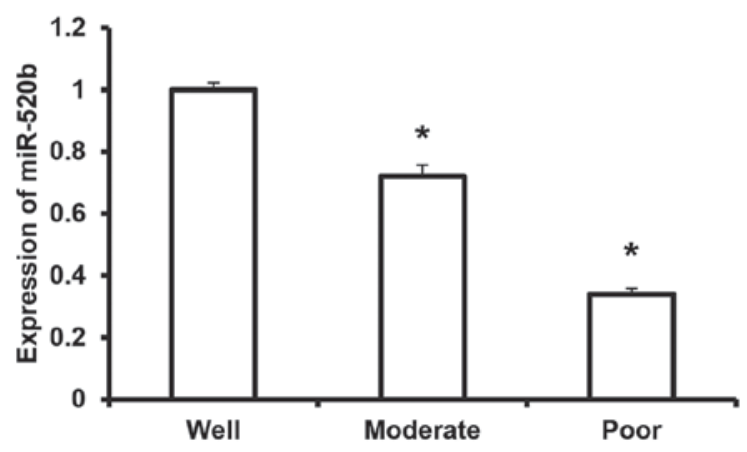

Figure 1. Expression levels of miR-520b in NSCLC tissues. Reverse transcription-quantitative polymerase chain reaction was performed to detect the expression levels of miR-520b in (A) NSCLC tissues and adjacent tissues, as well as based on different subgroup analysis $\left({ }^{* *} \mathrm{P}<0.01\right.$ vs. adjacent tissues), including (B) with or without lymph node metastasis ("P<0.05 vs. N0), (C) TNM staging ( $\mathrm{P}<0.05$ vs. I/II) and (D) differentiation status ("P<0.05 vs. the well group). miR, microRNA; NSCLC, non-small cell lung cancer. N0, with no lymph node metastasis; N1, with lymph node metastasis.

observed under light microscope and 5 fields were randomly selected under high magnification, and the cells infiltrating through the membrane were counted, based on which the cell invasion and migration abilities were assessed.

Flow cytometry. At 48 h following transfection, cells were digested with trypsin and rinsed twice with pre-cold PBS. Cell cycle was detected by flow cytometry, using a Cell Cycle Assay kit (BD Biosciences, Franklin Lakes, NJ, USA), according to the manufacturer's instructions. Cells $\left(2 \times 10^{6}\right.$ cells $\left./ \mathrm{ml}\right)$ were incubated with $200 \mu \mathrm{l}$ solution A at room temperature for $10 \mathrm{~min}$, and then incubated with $150 \mu \mathrm{l}$ solution $\mathrm{B}$ at room temperature for $10 \mathrm{~min}$, followed by incubation at room temperature with $120 \mu$ l solution $\mathrm{C}$ in the dark for $10 \mathrm{~min}$. Fluorescence was detected using a flow cytometer, and the results were analyzed with Modfit software (version 3.2; Verity Software House, Inc., Topsham, ME, USA).

Western blot analysis. Cells were lysed with RIPA lysis buffer (containing 1\% phenylmethane sulfonyl fluoride) on ice for $40 \mathrm{~min}$. Following centrifugation at $4^{\circ} \mathrm{C}$ at $10,000 \times \mathrm{g}$ for $10 \mathrm{~min}$, the supernatant was collected. Following protein concentration determination using the BCA method (Beyotime Institute of Biotechnology), $10 \mu \mathrm{l}$ protein samples were subjected to $10 \%$ SDS-PAGE, and electronically transferred onto a polyvinylidene difluoride membrane. Following blocking with $50 \mathrm{~g} / \mathrm{l}$ non-fat milk at room temperature for $1 \mathrm{~h}$, the membrane was incubated with rabbit anti-human anti-Rab22A polyclonal primary antibody (1:1,000; ab99205; Abcam, Cambridge, UK), rabbit anti-human
anti-E-cadherin primary antibody (1:1,000; Beyotime Institute of Biotechnology), rabbit anti-human anti-vimentin primary antibody (1:1,000; Beyotime Institute of Biotechnology), and mouse anti-human anti-GAPDH primary antibody (1:4,000; Abcam), respectively, at $4^{\circ} \mathrm{C}$ overnight. Following washing with TBS-Tween-20 three times, the membrane was incubated with horseradish peroxidase-conjugated goat anti-mouse $(1: 4,000 ; \mathrm{A} 0208)$ or goat anti-rabbit $(1: 2,000 ; \mathrm{A} 0216$; both Beyotime Institute of Biotechnology) secondary antibodies at room temperature for $1 \mathrm{~h}$. Colorization was performed using BeyoECL Plus (Beyotime Institute of Biotechnology).

Dual-luciferase reporter assay. According to bioinformatics prediction (Targetscan 7.1; targetscan.org/vert_72/), the wild-type and mutant seed regions of Rab22A 3'-UTR for miR-520b were added with Spe-1 and HindIII restriction sites, respectively, which were then cloned into the pMIR-REPORT luciferase reporter plasmid (Thermo Fisher Scientific, Inc.). A total of $0.5 \mu \mathrm{g}$ plasmid containing wild-type or mutant 3'-UTR DNA sequence was transfected into HEK293T cells (Type Culture Collection of the Chinese Academy of Sciences) with Lipofectamine $2000{ }^{\circledR}$ (Thermo Fisher Scientific, Inc.), together with the miR-520b mimics. Following $24 \mathrm{~h}$, the cells were collected and lysed with a Dual-Luciferase ${ }^{\circledR}$ Reporter Assay system (Promega Corporation, Madison, WI, USA), and the luciferase was detected with a GloMax 20/20 luminometer. Renilla was used as an internal reference.

Statistical analysis. Data were expressed as mean \pm standard deviation. Statistical analysis was performed with SPSS 17.0 software (SPSS, Inc., Chicago, IL, USA). Student's t-test was 
A

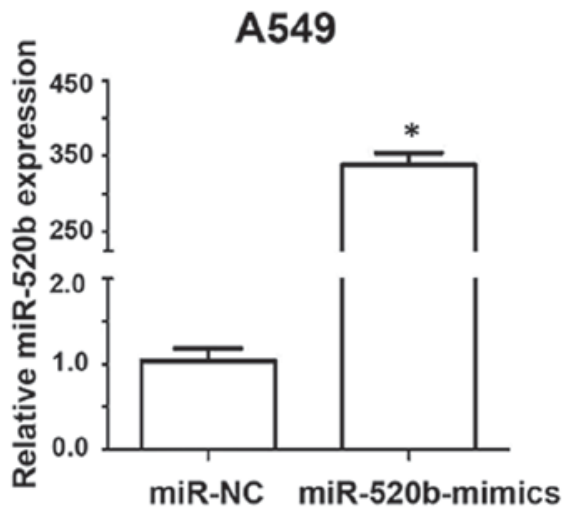

B

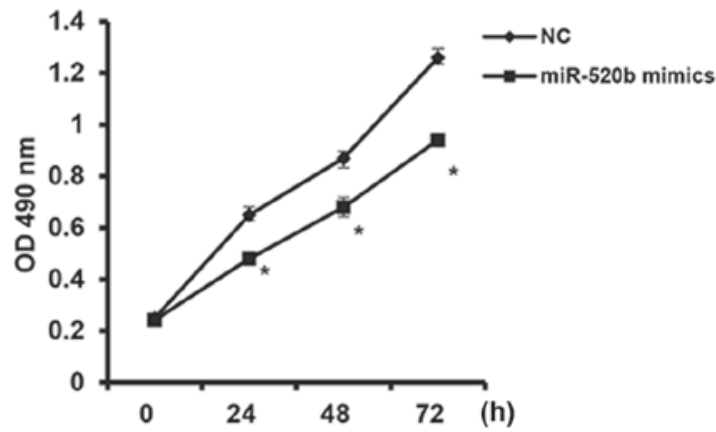

C

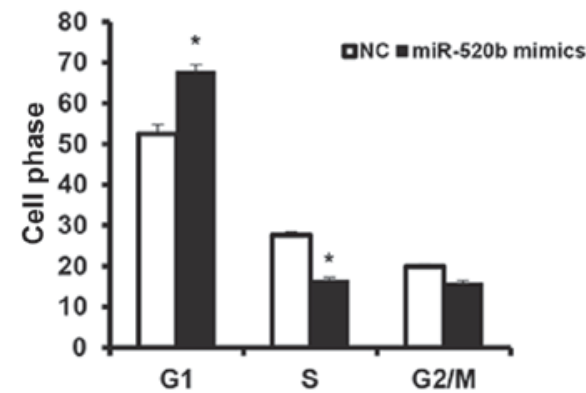

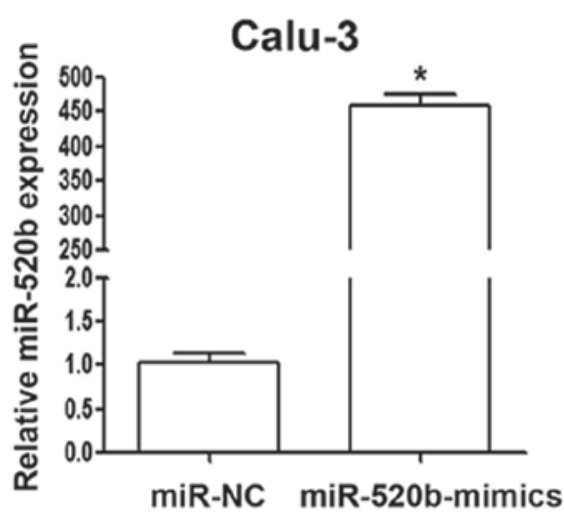
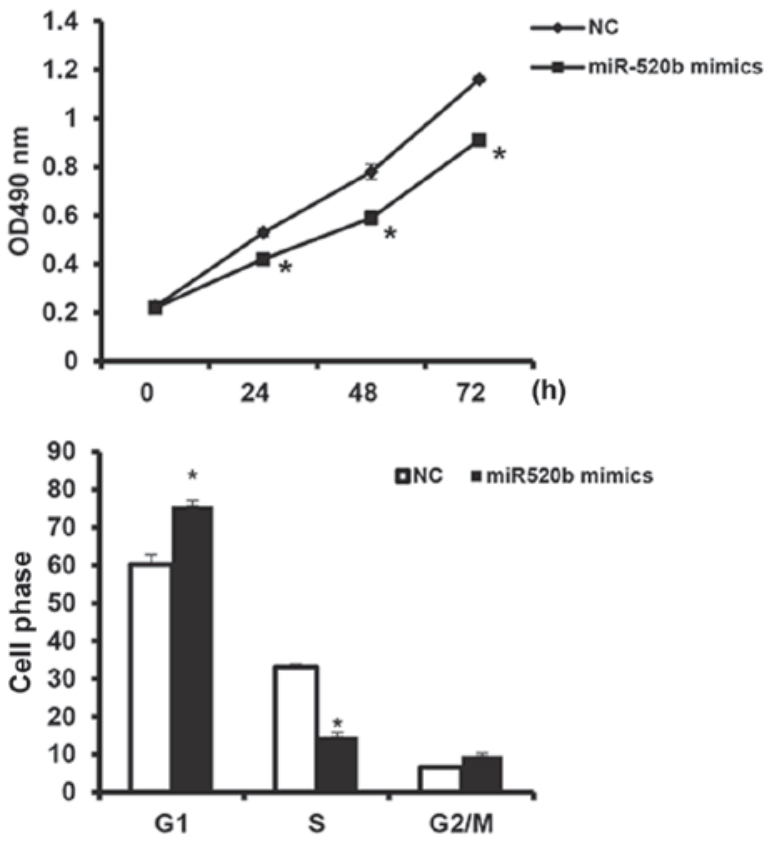

Figure 2. Effects of miR-520b on proliferation of NSCLC cells. NSCLC A549 and Calu-3 cells were transfected with miR-520b mimics. (A) The relative expression levels of miR-520b were detected with Reverse transcription-quantitative polymerase chain reaction. (B) Cell proliferation at indicated time points was detected with the cell counting kit-8 assay. (C) Cell cycle was detected with flow cytometry. "P<0.05 vs. NC. miR, microRNA; NSCLC, non-small cell lung cancer; NC, negative control; OD, optical density.

used for comparison between two groups. One-way analysis of variance was used for comparisons between multiple groups, followed by the Student-Newman-Keuls method as a post hoc test. $\mathrm{P}<0.05$ was considered to indicate a statistically significant difference.

\section{Results}

Expression of miR-520b in NSCLC tissue. To investigate the expression of miR-520b in NSCLC tissue and its clinical significance, RT-qPCR was performed. The results demonstrated that the expression level of miR-520b was significantly decreased in NSCLC tissue $(0.24 \pm 0.04)$ compared with adjacent tissue $(\mathrm{P}<0.01$; Fig. 1A). Furthermore, the expression level of miR-520b in the melanoma tissue from the N1 group $(0.47 \pm 0.06)$ was significantly lower than that in the N0 group $(\mathrm{P}<0.05)$ (Fig. 1B). According to the TNM staging, the expression level of miR-520b in the tumors at stage III $(0.62 \pm 0.07)$ was significantly lower than that of the tumors at stages I/II $(\mathrm{P}<0.05)$ (Fig. 1C). The expression levels of miR-520b for the moderate- and poor-differentiation groups were significantly lower than the well-differentiation group $(\mathrm{P}<0.05$; Fig. 1D). These results suggest that the expression of miR-520b is downregulated in NSCLC tissue, which may be closely associated with tumor invasion and metastasis, and disease pathogenesis.

Effects of miR-520b on proliferation of NSCLC cells. To investigate the effects of miR-520b on the proliferation of NSCLC cells, the CCK- 8 assay was performed. The results revealed that following transfection, the miR-520b expression levels were significantly elevated in the A549 and Calu-3 cells (Fig. 2A). The findings demonstrated that, following transfection with miR-520b mimics, the $\mathrm{OD}_{490 \mathrm{~nm}}$ values of A549 and Calu-3 cells at 24,48 , and $72 \mathrm{~h}$ post-transfection were significantly lower than the $\mathrm{NC}$ group (all $\mathrm{P}<0.05$; Fig. 2B). Cell cycle stage was detected by flow cytometry. The results demonstrated that, the number of miR-520b-transfected cells in the $G_{1}$ phase was significantly elevated, whereas the miR-520b-transfected cells in the $\mathrm{S}$ phase was significantly 
A
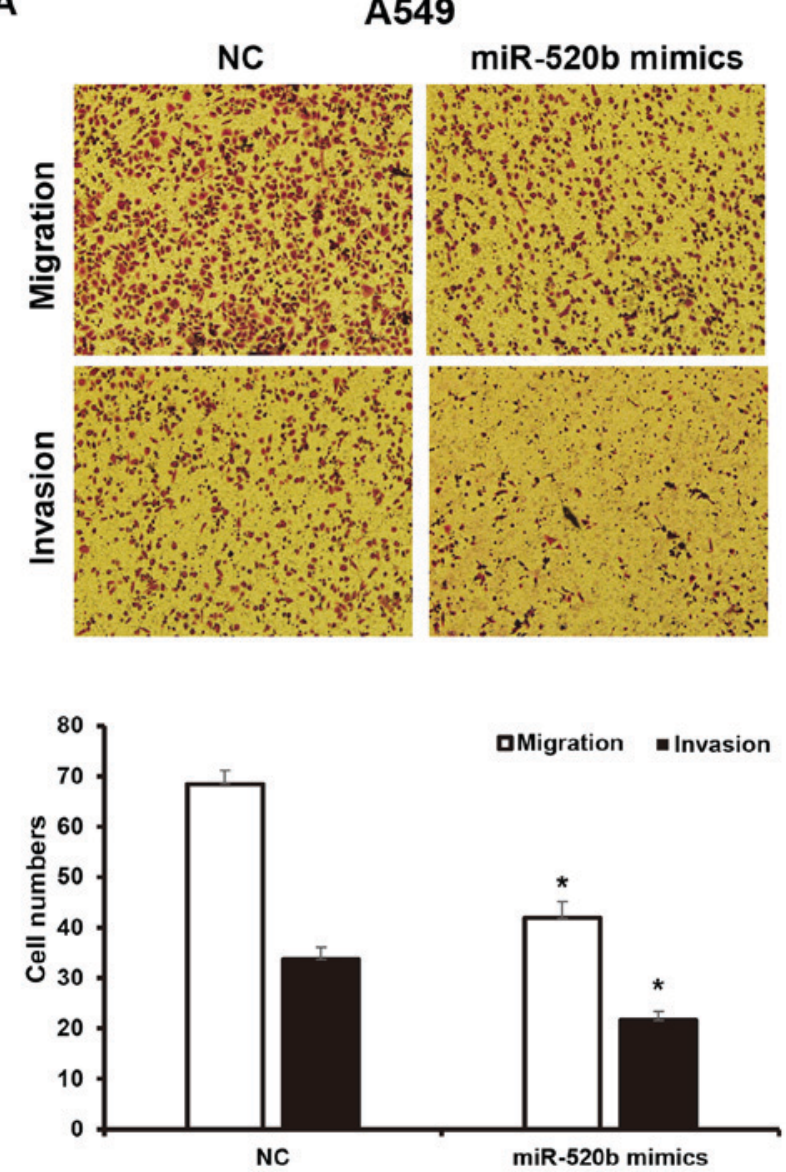

B
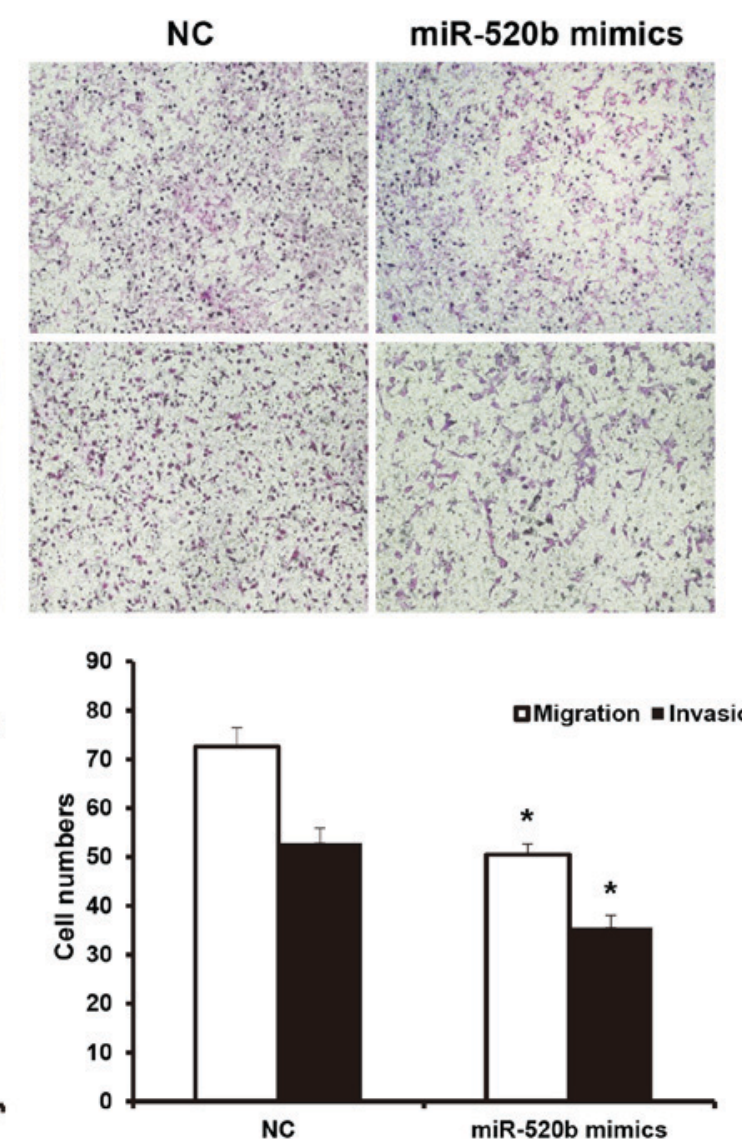

Figure 3. Effects of miR-520b on invasion and migration abilities of NSCLC cells. Following transfection with miR-520b mimics, the invasion and migration abilities of NSCLC (A) A549 and (B) Calu-3 cells were assessed with the Transwell chamber assay (magnification, x100). * $<<0.05$ vs. NC. miR, microRNA; NSCLC, non-small cell lung cancer; NC, normal control.

reduced, indicating $\mathrm{G}_{1} / \mathrm{S}$ phase arrest of these cells following miR-520b transfection (Fig. 2C). These results suggest that miR-520b inhibited the proliferation of NSCLC cells in vitro, the over-expression of which may be one of the reasons for the recurrence and metastasis of NSCLC.

Effects of miR-520b on invasion and migration abilities of NSCLC cells. To investigate the invasion and migration abilities of NSCLC cells, a Transwell chamber assay was performed. The results demonstrated that, following transfection with miR-520b, the migration ability of NSCLC cells was significantly inhibited. The numbers of A 549 and Calu-3 cells crossing the chamber membrane were significantly lower than the NC groups $(41.9 \pm 3.2$ vs. $68.3 \pm 2.8$, and $50.5 \pm 2.1$ vs. $72.6 \pm 3.8$ respectively; $\mathrm{P}<0.05)$. Results from the invasion test demonstrated that the number of transfected A549 cells crossing the Transwell chamber membrane $(21.6 \pm 1.8)$ was significantly lower than the NC group (33.7 \pm 2.4$)$. Furthermore, the number of the transfected Calu-3 cells crossing the Transwell chamber membrane $(35.6 \pm 2.5)$ was significantly lower than the $\mathrm{NC}$ group (52.8 $\pm 3.1 ; \mathrm{P}<0.05$; Fig. $3 \mathrm{~A}$ and $\mathrm{B})$. These results suggest that miR-520b may inhibit the invasion and metastasis abilities of NSCLC cells.

Effects of miR-520b on epithelial mesenchymal transformation (EMT) of NSCLC. To investigate the effects of miR-520b on EMT of NSCLC, expression levels of E-cadherin (an EMT marker) were detected by western blot analysis. The results demonstrated that, following transfection with miR-520 mimics, the expression levels of E-cadherin were markedly elevated, whereas the expression levels of vimentin were markedly declined in the A549 cells (Fig. 4A). Similar results and expression trends were observed in Calu-3 cells although the changes were minimal (Fig. 4B). These results suggest that miR-520b may inhibit the EMT of NSCLC.

Rab22A is a direct target for miR-520b. miRNAs target the 3'-UTR of mRNAs to exert biological functions. Therefore, the target for miR-520b was investigated to further explore its underlying mechanism. Through the mRNA target gene prediction, using Targetscan7.1 software (targetscan.org), the tumor suppressor gene Rab22A was recognized as the potential target gene for miR-520b. The results from western blot analysis demonstrated that the expression levels of Rab22A in the NSCLC cells transfected with miR-520b mimics were markedly downregulated. Dual luciferase reporter assay results demonstrated that, the fluorescence value in the wild-type group was significantly lower than the control group $(\mathrm{P}<0.05)$, whereas no significant differences were observed between the mutant and $\mathrm{NC}$ groups (Fig. 5A and B). These results suggested that $\mathrm{miR}-520 \mathrm{~b}$ could directly regulate the expression of Rab22A. 
A

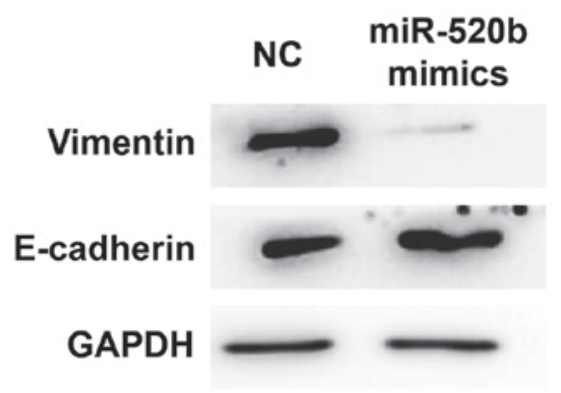

B Calu-3

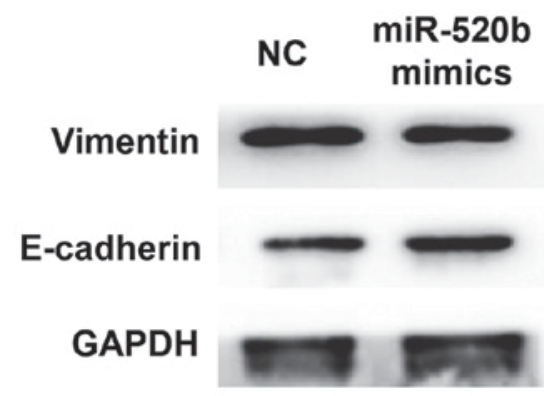

Figure 4. Effects of miR-520b on epithelial mesenchymal transformation of non-small cell lung cancer cells. Following transfection with miR-520b mimics, expression levels of E-cadherin and vimentin in (A) A549 and (B) Calu-3 cells were assessed via western blot analysis. miR, microRNA; NC, normal control.

miR-520b regulates proliferation and metastasis of NSCLC through targeting on Rab22A. To further investigate the effects of miR-520b regulating Rab22A on NSCLC cell functions, these cells were transfected with Rab22A-over-expressed lentivirus (western blot analysis confirmed the successful overexpression of Rab22A in the transfected cells, as presented in Fig. 6A), and the reversal effects of Rab22A on the miR-520b mimics-transfected cell functions were analyzed. In addition, compared with the miR-520b mimics group, Rab22A expression was significantly elevated in the reversal group (Fig. 6B). The results demonstrated that, compared with miR-520b mimics, the $\mathrm{OD}_{490 \mathrm{~nm}}$ values at 24, 48 and $72 \mathrm{~h}$ in the reversal group were significantly elevated $(\mathrm{P}<0.05$; Fig. 6C). Cell cycle analysis demonstrated that, compared with the miR-520b mimics group, the cell number in the $G_{1}$ phase was significantly higher, whereas the cell number in the $S$ phase was significantly lower, in the reversal group, indicating accelerated $G_{1} / S$ phase conversion (Fig. 6D). Results from Transwell chamber assay demonstrated that the cell numbers of migration and invasion in the Rab22A reversal group were both significantly higher than the miR-520b mimics group, indicating enhanced invasion and metastasis abilities (Fig. 6E). These results suggest that Rab22A could reverse the miR-520b-induced inhibiting effects on proliferation and metastasis of NSCLC.

\section{Discussion}

Tumor recurrence and metastasis represent key factors limiting the clinical treatment efficacy of NSCLC, the underlying mechanism of which remains to be elucidated (7). A number of previous studies have demonstrated that the recurrence and metastasis of NSCLC are closely associated with abnormal gene expression, methylation, gene mutation, drug resistance and immunosuppression $(24,25)$. miRNA molecules are associated with almost all pathophysiological biological processes, which provides rationale for their use in early diagnosis and target therapy for tumors (26). A number of miRNAs are associated with the development of NSCLC (27). In the present study, it was demonstrated that miR-520b expression was significantly downregulated in NSCLC tissue, which was negatively correlated with lymph node metastasis and TNM staging. Cellular and molecular experiments confirmed that miR-520b targeted Rab22A to regulate the proliferation and metastasis of NSCLC.
A
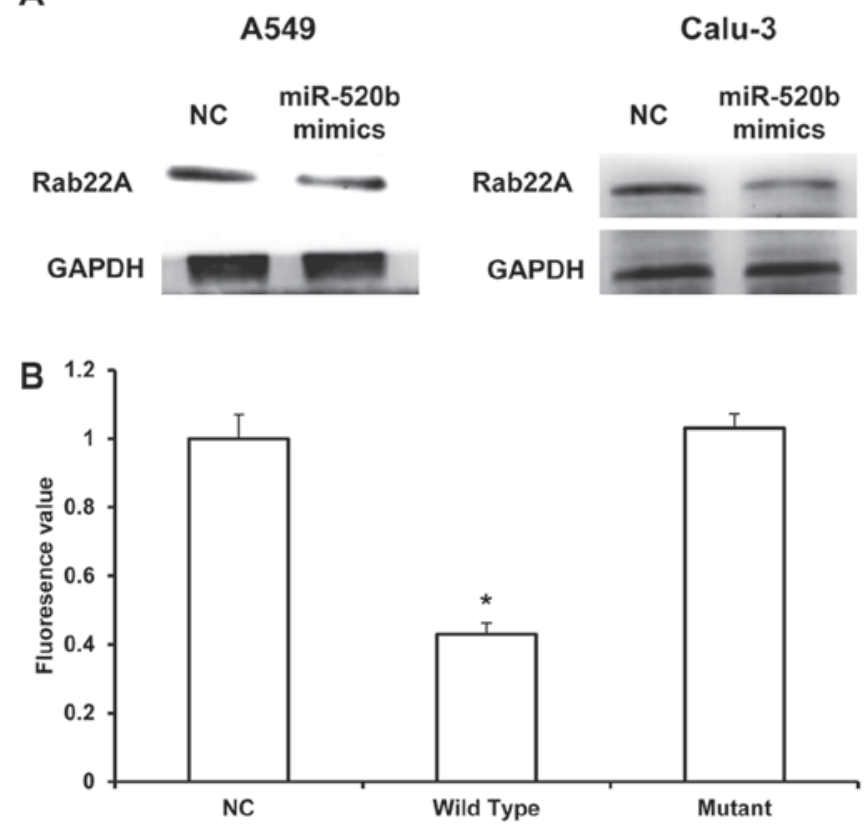

Figure 5. miR-520b directly targets Rab22A. (A) Following transfection with miR-520b mimics, expression levels of Rab22A in A549 and Calu-3 cells were assessed with Western blot analysis. (B) Dual luciferase reporter assay was performed to evaluate the direct interaction between miR-520b and Rab22A. ${ }^{*} \mathrm{P}<0.05$ vs. NC. miR, microRNA; NC, normal control.

miRNAs are a class of post-transcriptional regulators that have attracted research attention in recent years, which are associated with the regulation of development in various diseases (28). Studies have demonstrated that various miRNAs serve important roles as oncogenes or tumor suppressor genes in tumor development, and that their abnormal expression may induce intracellular oncogene/tumor suppressor gene imbalance, thereby promoting cell carcinogenesis, and tumor recurrence and metastasis (29). It has been indicated that miR-26a inhibits the proliferation, invasion and metastasis of malignant melanoma by targeting microphthalmia-associtated transcription factor genes (30). Furthermore, miR-125b promotes the sensitivity of nasopharyngeal carcinoma to radiotherapy via regulating the A20/nuclear factor (NF)- $\mathrm{B}$ signaling pathway (31). Ren et al (32) have recently demonstrated that miR-210-3p promotes EMT and bone metastasis of pancreatic cancer 
A

$$
\text { NC } \begin{gathered}
\text { Rab22A } \\
\text { over-expression }
\end{gathered}
$$

Rab22A

GAPDH

B

\section{A549}

Calu-3

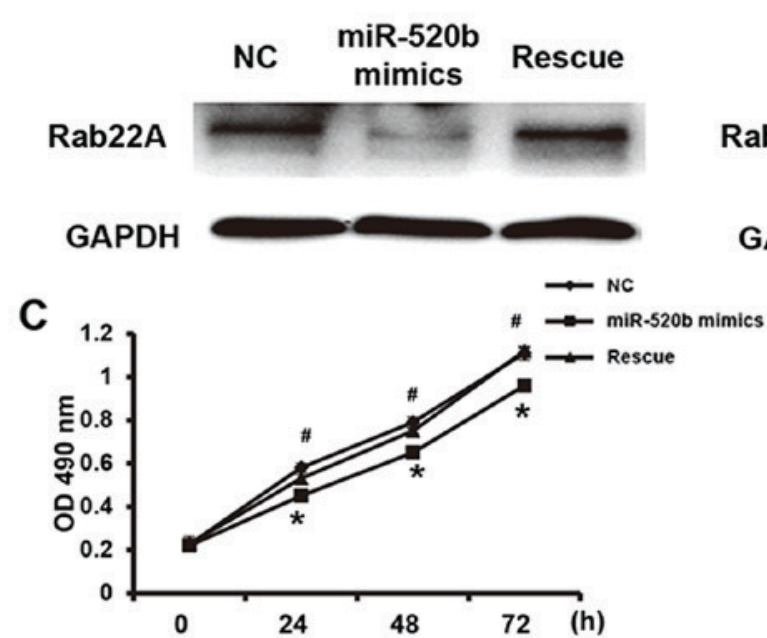

Rab22A

NC miR-520b $\begin{gathered}\text { mimics } \\ \text { Rescue }\end{gathered}$

D

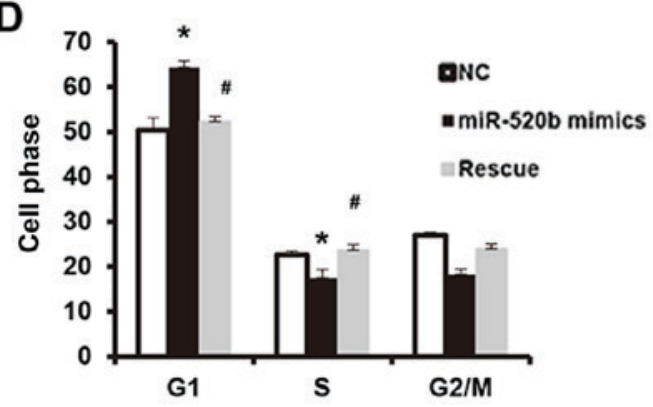

E

A549
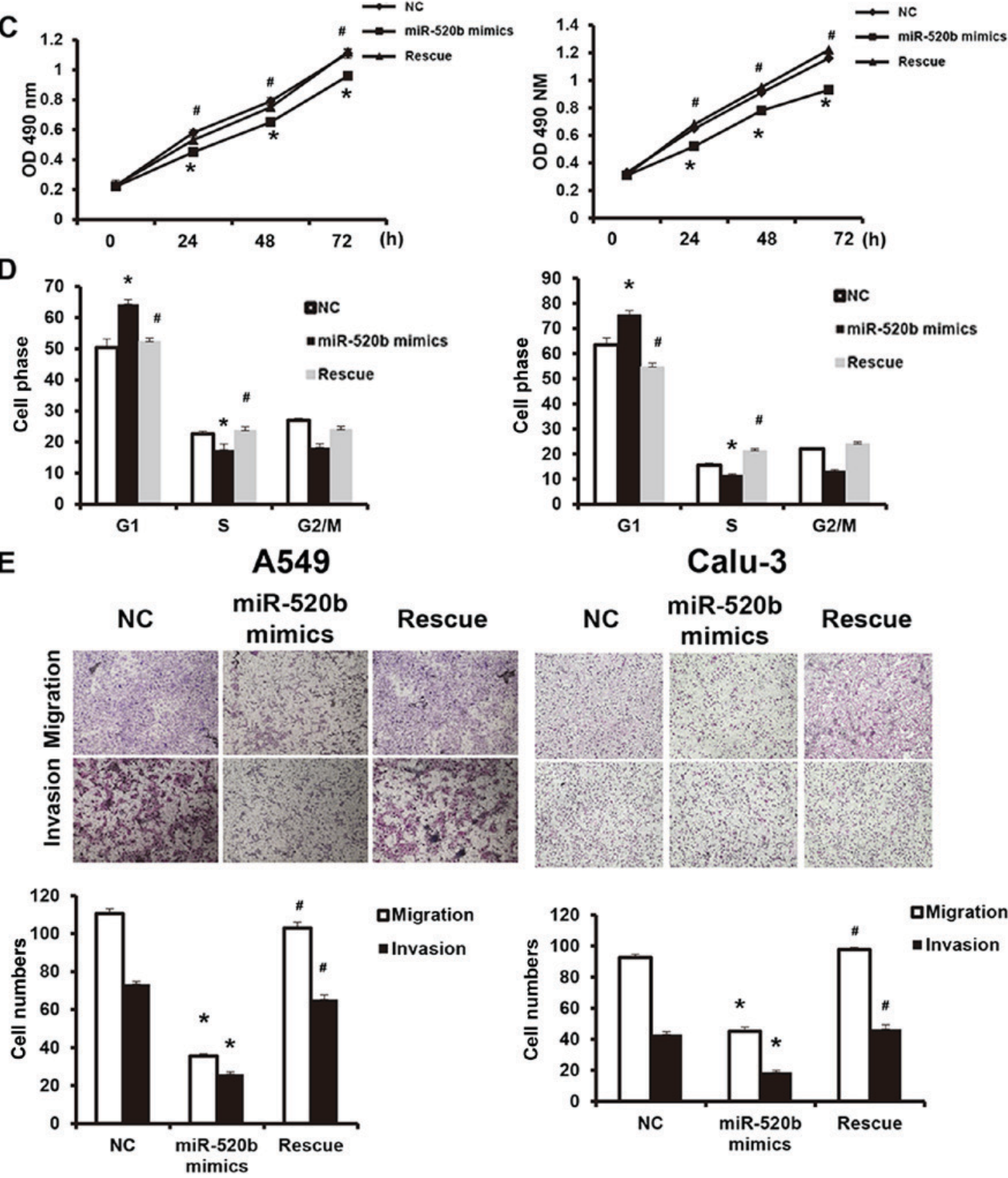

Figure 6. miR-520b regulates proliferation and metastasis of non-small cell lung cancer through targeting Rab22A. The reversal effects of Rab22A on miR-520b mimic-transfected cell functions were analyzed. (A) A549 cells overexpressed Rab22A. (B) Expression levels of Rab22A were detected by western blot analysis. (C) Cell proliferation was assessed via a cell counting kit-8 assay. (D) Cell cycle was detected with flow cytometry. (E) Cell invasion and migration abilities were assessed with Transwell chamber assay (magnification, x100). ${ }^{*} \mathrm{P}<0.05$ vs. NC; ${ }^{*} \mathrm{P}<0.05$ vs. miR-520b mimics. miR, microRNA; NC, normal control; OD, optical density. 
through the regulation of NF- $\mathrm{NB}$ signaling pathway. In addition, due to the disturbed expression profiles of mRNA in both tumor tissues and cells, the same miRNA may target different genes to exert biological functions in different tumors (33). miR-503 has been demonstrated to target and regulate the CyclinD1 gene in esophageal squamous cell carcinoma (34), and targets B cell lymphoma-2 in the hepatocellular carcinoma (35). miR-520b is a newly discovered tumor-associated miRNA molecule, which acts as a tumor suppressor in a variety of tumors. It has been demonstrated that miR-520b inhibits the stemness of head and neck tumors via targeting cluster of differentiation (CD)44 expression (36). Furthermore, miR-520b may target defective in cullin neddylation 1 domain containing 1 expression in colon cancer and inhibit tumor development (37).

The present results demonstrated that the expression of miR-520b was significantly downregulated in NSCLC tissues, which was negatively associated with lymph node metastasis and TNM staging. Results from in vitro experiments demonstrated that the upregulation of miR-520b inhibited the proliferation and metastasis of NSCLC A549 and Calu-3 cells. These results indicate that miR-520b may serve a role as a tumor suppressor in the development of NSCLC. Further in-depth studies are required to investigate the association between miR-520b and disease outcomes. Rab22A is a small molecule GTP binding protein, or small molecule GTPase, which belongs to the Ras superfamily $(38,39)$. Rab22A is generally activated following binding to GTP in transit vesicles. GTP is converted to GDP following energy dissipation, and Rab22A is also inactive (39). A large number of studies have demonstrated that Rab22A exerts oncogene function in the development of various tumors (40). $\mathrm{Su}$ et al (41) have recently demonstrated that Rab22A is highly expressed in melanoma and promotes tumor proliferation and metastasis (41). Furthermore, Zhou et al (42) have suggested that Rab22A promotes the metastasis of lung cancer cells by regulating the recycling of CD147. In the present study, bioinformatics prediction demonstrated that miR-520b may be associated with the regulation of Rab22A expression. The results from western blot analysis demonstrated that upregulation of miR-520b could downregulate the expression of Rab22A. Conversely, dual luciferase reporter assay confirmed that Rab22A was the target gene for miR-520b. In order to further verify that miR-520b exerted biological functions though Rab22A, the expression of Rab22A was reversed, which inhibited the tumor suppressing function of miR-520b in NSCLC. These findings suggest that miR-520b exerts biological functions through regulating Rab22A. Although these results demonstrated that Rab22A was the target of miR-520b, the underlying mechanisms for cell proliferation, invasion and migration are yet to be elucidated.

In conclusion, the present results demonstrated that miR-520b served a role as a tumor suppressor gene in the development of NSCLC. The downregulated expression of miR-520b in lung cancer tissue was closely associated with tumor development. miR-520b targeted Rab22A to exert the tumor suppressing function.

\section{Acknowledgements}

The authors are grateful to Dr Hua Hu, Thoracic Hospital of Shandong Province (Jinan, China) for providing assistance with project support and manuscript preparation.

\section{Funding}

No funding received.

\section{Availability of data and materials}

The datasets used and/or analyzed during the current study are available from the corresponding author on reasonable request.

\section{Authors' contributions}

LZ and SY designed the study, performed the experiments, collected and analyzed the data, and wrote the manuscript.

\section{Ethics approval and consent to participate}

Prior written and informed consent was obtained from every patient and the study present was approved by the Ethics Review Board of Shandong Chest Hospital.

\section{Patient consent for publication}

Prior written and informed consent was obtained from every patient.

\section{Competing interests}

The authors declare that they have no competing interests.

\section{References}

1. Gu Y, Lv H, Zhao J, Li Q, Mu G, Li J, Wuyang J, Lou G, Wang R, Zhang $Y$ and Huang $X$ : Influence of the number and interval of treatment cycles on cytokine-induced killer cells and their adjuvant therapeutic effects in advanced non-small-cell lung cancer (NSCLC). Int Immunopharmacol 50: 263-269, 2017.

2. Lamb YN and Scott LJ: Osimertinib: A review in T790M-positive advanced non-small cell lung cancer. Target Oncol 12: 555-562, 2017.

3. Powell AC, Mirhadi AJ, Loy BA, Happe LE, Long JW, Kren EM and Gupta AK: Presentation at computed tomography (CT) scan of the thorax and first year diagnostic and treatment utilization among patients diagnosed with lung cancer. PLoS One 12: e0181319, 2017.

4. He J, Yu L, Wang CM and Zhou XF: MiR-1275 promotes non-small cell lung cancer cell proliferation and metastasis by regulating LZTS3 expression. Eur Rev Med Pharmacol Sci 22: 2680-2687, 2018.

5. Chae YK, Arya A, Iams W, Cruz MR, Chandra S, Choi J and Giles F: Current landscape and future of dual anti-CTLA4 and PD-1/PD-L1 blockade immunotherapy in cancer; lessons learned from clinical trials with melanoma and non-small cell lung cancer (NSCLC). J Immunother Cancer 6: 39, 2018.

6. Proto C, Lo Russo G, Corrao G, Ganzinelli M, Facchinetti F, Minari R, Tiseo $\mathbf{M}$ and Garassino MC: Treatment in EGFR-mutated non-small cell lung cancer: How to block the receptor and overcome resistance mechanisms. Tumori 103: 325-337, 2017.

7. Botsa EI, Thanou IL, Papatheodoropoulou AT and Thanos LI: Thermal ablation in the management of adrenal metastasis originating from Non-small cell lung cancer: A 5-year Single-center experience. Chin Med J (Engl) 130: 2027-2032, 2017.

8. Zhang G, Zhang D, Wu J, Zhang F, Zhu Z, Chen K, Zhang N, Jin J, Feng J, Lin N, et al: Low serum levels of Pre-surgical total cholesterol are associated with unfavorable overall survival in patients with operable Non-small cell lung cancer. Clin Lab 64: 321-327, 2018. 
9. Camidge DR, Kim DW, Tiseo M, Langer CJ, Ahn MJ, Shaw AT, Huber RM, Hochmair MJ, Lee DH, Bazhenova LA, et al: Exploratory analysis of brigatinib activity in patients with anaplastic lymphoma Kinase-positive Non-small-cell lung cancer and brain metastases in two clinical trials. J Clin Oncol: JCO2017775841, 2018.

10. Xu Y, Zhang F, Pan X, Wang G, Zhu L, Zhang J, Wen D and Lu S: Xenograft tumors derived from malignant pleural effusion of the patients with non-small-cell lung cancer as models to explore drug resistance. Cancer Commun (Lond) 38: 19, 2018.

11. Zhou J, Yu Y, Pei Y, Cao C, Ding C, Wang D, Sun L and Niu G: A potential prognostic biomarker SPC24 promotes tumorigenesis and metastasis in lung cancer. Oncotarget 8: 65469-65480, 2017.

12. Ali A, Kim SH, Kim MJ, Choi MY, Kang SS, Cho GJ, Kim YS, Choi JY and Choi WS: O-GlcNAcylation of NF- $\kappa$ B promotes lung metastasis of cervical cancer cells via upregulation of CXCR4 expression. Mol Cells 40: 476-484, 2017.

13. Casaluce F, Sgambato A, Maione P, Sacco PC, Santabarbara G and Gridelli C: Selumetinib for the treatment of non-small cell lung cancer. Expert Opin Investig Drugs 26: 973-984, 2017.

14. Shlomi D, Abud M, Liran O, Bar J, Gai-Mor N, Ilouze M, Onn A, Ben-Nun A, Haick H and Peled N: Detection of lung cancer and EGFR mutation by electronic nose system. J Thorac Oncol 12: 1544-1551, 2017

15. Lee WY, Chen PC, Wu WS, Wu HC, Lan CH, Huang YH, Cheng $\mathrm{CH}$, Chen $\mathrm{KC}$ and Lin $\mathrm{CW}$ : Panobinostat sensitizes KRAS-mutant non-small-cell lung cancer to gefitinib by targeting TAZ. Int J Cancer 141: 1921-1931, 2017.

16. Ma W, Brodie S, Agersborg S, Funari VA and Albitar M: Significant improvement in detecting BRAF, KRAS, and EGFR mutations using Next-generation sequencing as compared with FDA-cleared kits. Mol Diagn Ther 21: 571-579, 2017.

17. Singh PK and Silakari O: Novel EGFR (T790M)-cMET dual inhibitors: Putative therapeutic agents for non-small-cell lung cancer. Future Med Chem 9: 469-483, 2017.

18. Wang H, Tang M, Ou L, Hou M, Feng T, Huang YE, Jin Y, Zhang H and Zuo G: Biological analysis of cancer specific microRNAs on function modeling in osteosarcoma. Sci Rep 7: 5382, 2017.

19. Wang L, Zhang B, Zheng W, Kang M, Chen Q, Qin W, Li C, Zhang Y, Shao Y and Wu Y: Exosomes derived from pancreatic cancer cells induce insulin resistance in $\mathrm{C} 2 \mathrm{C} 12$ myotube cells through the PI3K/Akt/FoxO1 pathway. Sci Rep 7: 5384, 2017.

20. Farooqi AA, Shu CW, Huang HW, Wang HR, Chang YT, Fayyaz S, Yuan SF, Tang JY and Chang HW: TRAIL, Wnt, sonic hedgehog, TGF $\beta$, and miRNA signalings are potential targets for oral cancer therapy. Int J Mol Sci 18: pii: E1523, 2017.

21. Li S, Zhang H, Ning T, Wang X, Liu R, Yang H, Han Y, Deng T, Zhou L, Zhang L, et al: MiR-520b/e regulates proliferation and migration by simultaneously targeting EGFR in gastric cancer. Cell Physiol Biochem 40: 1303-1315, 2016.

22. Yasukawa M, Kawaguchi T, Kawai N, Tojo T and Taniguchi S: Prognostic significance of mode of nodal involvement in pulmonary pN1 squamous cell carcinoma. Kyobu Geka 71: 163-168, 2018 (In Japanese).

23. Livak KJ and Schmittgen TD: Analysis of relative gene expression data using real-time quantitative PCR and the 2(-Delta Delta C(T)) method. Methods 25: 402-408, 2001.

24. Nia ES, Garland LL, Eshghi N, Nia BB, Avery RJ and Kuo PH: Incidence of brain metastases on follow-up ${ }^{18} \mathrm{~F}-\mathrm{FDG}$ PET/CT scans of non-small cell lung cancer patients: Should we include the brain? J Nucl Med Technol 45: 193-197, 2017.

25. Liang Y, Xu X, Wang T, Li Y, You W, Fu J, Liu Y, Jin S, Ji Q, Zhao W, et al: The EGFR/miR-338-3p/EYA2 axis controls breast tumor growth and lung metastasis. Cell Death Dis 8: e2928, 2017.

26. Lu W, Zhang H, Niu Y, Wu Y, Sun W, Li H, Kong J, Ding K, Shen HM, Wu H, et al: Long non-coding RNA linc00673 regulated non-small cell lung cancer proliferation, migration, invasion and epithelial mesenchymal transition by sponging miR-150-5p Mol Cancer 16: 118, 2017.
27. Yuwen DL, Sheng BB, Liu J, Wenyu W and Shu YQ: MiR-146a-5p level in serum exosomes predicts therapeutic effect of cisplatin in non-small cell lung cancer. Eur Rev Med Pharmacol Sci 21: 2650-2658, 2017

28. Lu Y, Tang L, Zhang Q, Zhang Z and Wei W: MicroRNA-613 inhibits the progression of gastric cancer by targeting CDK9. Artif Cells Nanomed Biotechnol 46: 980-984, 2018.

29. Moles R: MicroRNAs-based therapy: A novel and promising strategy for cancer treatment. Microrna 6: 102-109, 2017.

30. Qian H, Yang C and Yang Y: MicroRNA-26a inhibits the growth and invasiveness of malignant melanoma and directly targets on MITF gene. Cell Death Discov 3: 17028, 2017.

31. Zheng Z, Qu JQ, Yi HM, Ye X, Huang W, Xiao T, Li JY, Wang YY, Feng J, Zhu JF, et al: MiR-125b regulates proliferation and apoptosis of nasopharyngeal carcinoma by targeting A20/NF-kappaB signaling pathway. Cell Death Dis 8: e2855, 2017.

32. Ren D, Yang Q, Dai Y, Guo W, Du H, Song L and Peng X: Oncogenic miR-210-3p promotes prostate cancer cell EMT and bone metastasis via NF- $\mathrm{KB}$ signaling pathway. Mol Cancer 16: $117,2017$.

33. Muralimanoharan S, Guo C, Myatt L and Maloyan A: Sexual dimorphism in miR-210 expression and mitochondrial dysfunction in the placenta with maternal obesity. Int J Obes (Lond) 39: $1274-1281,2015$

34. Jiang L, Zhao Z, Zheng L, Xue L, Zhan Q and Song Y: Downregulation of miR-503 promotes ESCC cell proliferation, migration, and invasion by targeting cyclin D1. Genomics Proteomics Bioinformatics 15: 208-217, 2017.

35. Yang X, Yin J, Xiang Q, Xie H, Yu J, Gan R and Lei X: MiR-503 sensitizes human hepatocellular carcinoma cells to cisplatin by targeting bcl-2. Zhong Nan Da Xue Xue Bao Yi Xue Ban 42: 605-610,2017 (In Chinese).

36. Lu YC, Cheng AJ, Lee LY, You GR, Li YL, Chen HY and Chang JT: MiR-520b as a novel molecular target for suppressing stemness phenotype of head-neck cancer by inhibiting CD44. Sci Rep 7: 2042, 2017.

37. Xiao J, Li G, Zhou J, Wang S, Liu D, Shu G, Zhou J and Ren F: MicroRNA-520b functions as a tumor suppressor in colorectal cancer by inhibiting DCUN1D1. Oncol Res: May 4, 2017 (Epub ahead of print).

38. Xiong F, Liu K, Zhang F, Sha K, Wang X, Guo X and Huang N: MiR-204 inhibits the proliferation and invasion of renal cell carcinoma by inhibiting RAB22A expression. Oncol Rep 35: 3000-3008, 2016

39. Yang Z, He M, Wang K, Sun G, Tang L and Xu Z: Tumor suppressive microRNA-193b promotes breast cancer progression via targeting DNAJC13 and RAB22A. Int J Clin Exp Pathol 7: 7563-7570, 2014

40. Wang T, Gilkes DM, Takano N, Xiang L, Luo W, Bishop CJ, Chaturvedi P, Green JJ and Semenza GL: Hypoxia-inducible factors and RAB22A mediate formation of microvesicles that stimulate breast cancer invasion and metastasis. Proc Natl Acad Sci USA 111: E3234-E3242, 2014.

41. Su F, Chen Y, Zhu S, Li F, Zhao S, Wu L, Chen X and Su J: RAB22A overexpression promotes the tumor growth of melanoma. Oncotarget 7: 71744-71753, 2016.

42. Zhou Y, Wu B, Li JH, Nan G, Jiang JL and Chen ZN: Rab22a enhances CD147 recycling and is required for lung cancer cell migration and invasion. Exp Cell Res 357: 9-16, 2017.

This work is licensed under a Creative Commons Attribution-NonCommercial-NoDerivatives 4.0 International (CC BY-NC-ND 4.0) License. 